\title{
Power allocation and transmitter switching for broadcasting with multiple energy harvesting transmitters
}

\author{
Hongbin Chen ${ }^{1 *}$, Feng Zhao ${ }^{1}$, Rong $\mathrm{Yu}^{2}$ and Xiaohuan $\mathrm{Li}^{3}$
}

\begin{abstract}
With the advancement of battery technology, energy harvesting communication systems attracted great research attention in recent years. However, energy harvesting communication systems with multiple transmitters and multiple receivers have not been considered yet. In this paper, the problem of broadcasting in a communication system with multiple energy harvesting transmitters and multiple receivers is studied. First, regarding the transmitters as a 'whole transmitter,' the optimal total transmission power is obtained and an optimal power allocation policy is extended to our system setup, with the aim of minimizing the transmission completion time. Then, a simpler power allocation policy is developed to allocate the optimal total transmission power to the data transmissions. As transmitter switching can provide flexibility and robustness to an energy harvesting communication system, especially when a transmitter is broken or the energy harvested by a transmitter is insufficient, a transmitter switching policy is further developed to choose a suitable transmitter to work whenever necessary. The results show that the proposed power allocation policy performs close to the optimal one and outperforms some heuristic ones in terms of transmission completion time. Besides, the proposed transmitter switching policy outperforms some heuristic ones in terms of number of switches.
\end{abstract}

Keywords: Rechargeable wireless communications; Energy harvesting; Power allocation; Transmitter switching

\section{Introduction}

Recently, energy harvesting or rechargeable sensor networks emerge as a new paradigm of sensor networks, in which the nodes can harvest energy from nature [1-3]. Before this, sensor network nodes are powered by batteries with limited energy storage, which are hard to recharge or replace. Therefore, the key challenge is to save energy and prolong network lifetime while guaranteeing the application-specific performance [4]. In contrast, the harvested energy relaxes the energy constraint, thus extending network lifetime in energy harvesting sensor networks. However, the energy that can be harvested from the environment is unstable and varies over time. Hence, the harvested energy should be carefully utilized in order to maximize the utility of energy harvesting sensor networks.

\footnotetext{
*Correspondence: chbscut@163.com

1 Key Laboratory of Cognitive Radio and Information Processing, Guilin

University of Electronic Technology, Ministry of Education, Guilin 541004, China Full list of author information is available at the end of the article
}

A lot of excellent works on energy management in energy harvesting sensor networks have been done. For example, two-stage communication power management algorithms were proposed for maximizing the utility of energy harvesting sensors, considering the energy neutrality constraint, the fixed power loss effects of circuitry, and the battery inefficiency and its capacity [5]. Energy allocation over source acquisition/compression and transmission for a single energy harvesting sensor was addressed which guarantees minimum average distortion while ensuring stability of the queue connecting source and channel encoders [6]. Discounted cost Markov decision process and reinforcement learning algorithms were applied to find optimal energy management policies to maximize the performance of a single energy harvesting sensor [7]. Through modeling the ambient energy supply by a two-state Markov chain and assuming a finite battery capacity, low-complexity transmission policies were proposed for a wireless sensor powered by an energy harvesting device [8]. Conditions for balancing a node's expected energy consumption with its expected energy harvesting

\section{Springer}

(c) 2014 Chen et al.: licensee Springer. This is an Open Access article distributed under the terms of the Creative Commons

Attribution License (http://creativecommons.org/licenses/by/4.0), which permits unrestricted use, distribution, and reproduction in any medium, provided the original work is properly credited. 
capability in a uniformly formed wireless sensor network were derived [9]. A stochastic Markov chain framework was proposed to characterize the interplay between the battery discharge policy and the irreversible degradation of the storage capacity [10].

In addition, other energy harvesting communication systems were also investigated [11-19]. Specifically, many energy harvesting communication schemes have been designed toward the goal of minimizing the transmission completion time. For example, optimal packet scheduling in a point-to-point communication system was studied in $[20,21]$. The goal is to adjust the transmission rate according to the data arrival and harvested energy, such that the time by which all packets are delivered is minimized. Transmission powers were optimized for a broadcasting communication system with an energy harvesting transmitter $[2,22,23]$. The objective is to minimize the time by which all packets are sent to their destinations. In [24], this problem was further studied assuming a finite capacity battery. While [2,20-24] studied packet scheduling over the additive white Gaussian noise (AWGN) channel, [25] studied packet scheduling in a point-to-point communication system over fading channels. Except for the above representative works, the effects of multiple access channel, parallel and fading Gaussian broadcast channels, interference channel, time-varying channels, wireless energy transfer, and packet arrivals during transmission were also taken into account [26-31].

The earlier works [20-25] mainly considered energy harvesting communication systems with only one transmitter. However, nowadays many communication systems are equipped with more than one transmitter. Therefore, it is necessary to study energy harvesting communication systems with multiple transmitters. In [26], optimal packet scheduling in a multiple access communication system with two energy harvesting transmitters was investigated. In [27], a communication system with an energy harvesting transmitter over parallel and fading Gaussian broadcast channels was studied. In [28], an optimal power allocation policy for a communication system with two energy harvesting transmitters over an interference channel was proposed. These works shed light on energy harvesting communication systems with multiple transmitters, but did not consider transmitter switching. In our opinion, transmitter switching can provide flexibility and robustness to an energy harvesting communication system, especially when a transmitter is unable to send data or the energy harvested from the environment is insufficient for data transmission. If this happens, other neighboring transmitters can turn to work and help the transmitter to proceed data transmission. To make the transmitter switching effective and decrease the switching overhead, a well-designed policy is essential to choosing the suitable transmitter to work.
Motivated by the above fact and lying on the earlier works $[1,2,32]$, power allocation and transmitter switching for broadcasting in a communication system with multiple energy harvesting transmitters and multiple receivers are studied in this paper. Our target is to minimize the transmission completion time and to reduce the number of switches under the energy causality constraint. The contributions of this paper are summarized as follows: 1) The optimal total transmission power and the optimal power allocation policy in [2] are rebuilt in the communication system with multiple energy harvesting transmitters. 2) A new power allocation policy is proposed which performs close to the optimal one but is simpler. 3) A new transmitter switching policy is proposed for the communication system with multiple energy harvesting transmitters and multiple receivers, which is more complex than the communication systems we studied before [1,32].

The remainder of this paper is organized as follows. In Section 2, the energy harvesting communication system with multiple transmitters and multiple receivers is described. In Section 3, the power allocation and transmitter switching policies are elaborated. Simulation results are presented in Section 4, and some concluding remarks are given in Section 5 .

\section{Energy harvesting communication system model}

We consider an energy harvesting communication system with multiple transmitters and multiple receivers, as shown in Figure 1. There are $M$ energy harvesting transmitters $\mathrm{TX}_{1}, \mathrm{TX}_{2}, \mathrm{TX}_{3}, \ldots, \mathrm{TX}_{M}$ and $N$ receivers $\mathrm{RX}_{1}, \mathrm{RX}_{2}, \mathrm{RX}_{3}, \ldots, \mathrm{RX}_{N}$. The energies arriving to the transmitters $E_{1}, E_{2}, E_{3}, \ldots, E_{M}$ are stochastic (both the arriving time and the amount are random) and independent of each other, while the data $B_{1}, B_{2}, B_{3}, \ldots, B_{N}$ are broadcasted by the transmitters in turn. Here $B_{n}$ is the data to be sent to the receiver $\operatorname{RX}_{n}(n=1, \ldots, N)$. The energies arrive during the course of transmission while the data are given before transmission. For tractability, the arriving time and the amount of energies are assumed to be known at the beginning of transmission (offline). This system looks like a multi-input multi-output one. But we view the transmitters as a 'whole transmitter' [1] and focus on transmitter switching that can enhance flexibility and robustness of the system. The transmitters cooperate to send the data $B_{1}, B_{2}, B_{3}, \ldots, B_{N}$ to the corresponding receivers. Every time, one of the transmitters $\mathrm{TX}_{m}$ will be active to broadcast data to the receivers. A transmitter switching policy will be designed to choose a suitable transmitter to work when the current working transmitter uses up its energy. Note that synchronization among the transmitters will be coordinated by a central controller. Control information should be exchanged between the 


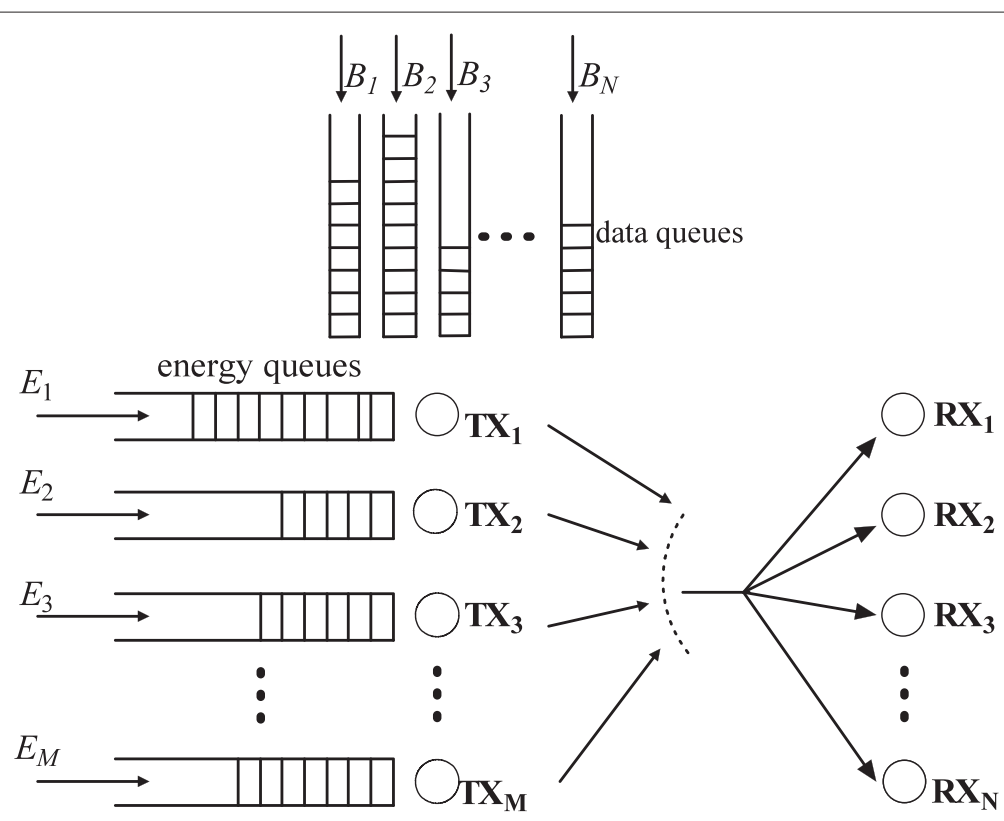

Figure 1 Energy harvesting communication system with multiple transmitters and multiple receivers.

transmitters and the controller periodically (the interval should be sufficiently small so that transmitter switching can be initiated in time). This may cause some delay, which is not considered in this work. When the controller decides that the data transmission should switch from one transmitter to another transmitter, the controller sends signaling information to the transmitters to invoke transmitter switching.

The energy arriving process for the transmitter $\mathrm{TX}_{m}$ is depicted in Figure 2. At the time $S_{m w}, w=1,2, \ldots$, the amount of energy $E_{m w}$ arrives to $\mathrm{TX}_{m} . E_{m 0}$ is the initial energy available in the battery of $\mathrm{TX}_{m}$ before the transmission starts. It is assumed that the batteries of the transmitters have infinite capacity and the harvested energy will not overflow.

Since there is only one transmitter sending data every time, the channel shown in Figure 1 is actually a broadcast channel. It is assumed that the chosen transmitter $\mathrm{TX}_{m}$ sends data to each receiver through an AWGN channel with different path losses. The signal received by the receiver $\mathrm{RX}_{n}$ can be represented by

$$
y_{m n}=h_{m n} x+v_{m n}, \quad m=1, \cdots, M ; n=1, \cdots, N
$$

where $h_{m n}$ is the path loss between $\mathrm{TX}_{m}$ and $\mathrm{RX}_{n}, x$ is the transmitted signal, and $v_{m n}$ is an AWGN with zero mean and variance $\sigma_{m n}^{2}$. Here $\sigma_{m n}^{2}=N_{m n} B_{o}$, where $N_{m n}$ is the noise power spectral density in the channel between $\mathrm{TX}_{m}$ and $\mathrm{RX}_{n}$, and $B_{o}$ is the bandwidth. It is assumed that all channels have the same bandwidth. Then, the capacity region for the broadcast channel is [2]

$$
\begin{aligned}
r_{m n} & \leq B_{o} \log _{2}\left(1+\frac{P_{n} h_{m n}}{\sum_{j<n} P_{j} h_{m n}+N_{m n} B_{o}}\right), \\
\sum_{n=1}^{N} P_{n} & =P_{o}
\end{aligned}
$$

where $r_{m n}$ is the transmission rate when $\mathrm{TX}_{m}$ sends data to $\mathrm{RX}_{n}$ with power $P_{n}, P_{n}$ is a portion of the total transmission power split to $\mathrm{RX}_{n}$, and $P_{o}$ is the total transmission power. In the following, we analyze data transmission from the information-theoretic point of view. It is assumed that data transmission is always successful no matter which transmitter broadcasts data. Moreover, the transmitters will not send data that has been sent out.

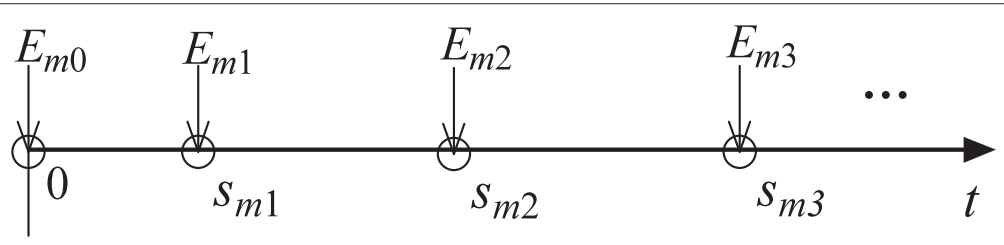

Figure 2 Energy arriving process for the transmitter $\mathrm{TX}_{m}$. 
To minimize the transmission completion time (by which the given number of bits are delivered to their intended receivers), power allocation should be executed among the data transmissions under the energy causality constraint. The energy causality means that at any given time, the total amount of consumed energy must be no more than the total amount of harvested energy. Following our previous work in [1], we treat all the transmitters as a whole transmitter (we only care about the amount of bits sent to the receivers while the bits sent by which transmitter do not matter) and find out the optimal total transmission power that achieves the maximum departure region [2] for a given deadline (the dual problem of transmission completion time minimization). Then, we rebuild the optimal power allocation policy [2] in our system setup. As the optimal power allocation policy needs the total transmission powers in all time slots to calculate the cutoff powers, we propose a simpler power allocation policy which only requires the total transmission power in the current time slot. Since every time only one transmitter is active to send data, transmitter switching is unavoidable. However, more switching among transmitters will bring greater control overhead, even though the energy consumed for transmitter switching is relatively small. To reduce control overhead and to save energy, the number of switches should be as least as possible. Following our previous work in $[1,32]$, we propose a transmitter switching policy to choose the suitable transmitter to send data with the principle of less number of switches. It should be emphasized that the turn of the working transmitters does not affect the transmission completion time. So we do power allocation first and then conduct transmitter switching. Note that with the optimal total transmission power at hand, transmitter switching can also be done before power allocation.

\section{Power allocation and transmitter switching policies}

In this section, the power allocation policies and the transmitter switching policy will be presented.

\subsection{Optimal total transmission power and optimal power allocation policy}

With the aim of minimizing the transmission completion time, the optimal total transmission power was obtained in [2]. Moreover, an optimal power allocation policy was derived for a broadcast communication system with an energy harvesting transmitter. Regarding the transmitters as a whole transmitter, we record the energy arriving to the transmitters in chronological order, as shown in Figure 3. Here $E_{0}$ is the sum of the initial energy in the batteries. The whole transmitter harvests energy at the time instant $s_{w}$ with amount $E_{w}$. This energy can be harvested by an arbitrary transmitter that we do not need to know.

With the new energy arriving process, the optimal total transmission power will be calculated and some properties of the optimal power allocation policy will be referenced in the following:

First, from Lemma 1, we know that the total transmission power remains constant between two consecutive energy harvesting instants, that is, the total transmission power only changes at an energy harvesting time instant.

Second, from Lemma 2, we get that the maximum departure region is a convex region. It means that there is one and only one optimal total transmission power.

Third, from Lemma 3, we derive the expression of the total transmission power as

$$
\begin{aligned}
i_{l} & =\arg \min _{i_{l-1}<w<W}\left\{\frac{\sum_{j=i_{l-1}}^{w-1} E_{j}}{s_{w}-s_{i_{l-1}}}\right\}, \\
P_{d l} & =\frac{\sum_{w=i_{l-1}}^{i_{l}-1} E_{w}}{s_{i_{l}}-s_{i_{l-1}}}
\end{aligned}
$$

where $s_{W}$ is the transmission completion time and the energy arriving time before it is denoted as $s_{W-1}$, and $P_{d l}$ is the optimal total transmission power for the whole transmitter $\mathrm{TX}_{d}$ over the interval $\left(s_{i_{l-1}}, s_{i_{l}}\right), l=1,2, \cdots$.

After calculating the optimal total transmission power, we further split the power to the data transmissions. Without loss of generality, we rank all of the variances from $\sigma_{d n}^{2}$ as $\sigma_{1}^{2} \leq \sigma_{2}^{2} \leq \cdots \leq \sigma_{N}^{2}$ and denote the receiver corresponding to $\sigma_{n}^{2}$ as the $n$th receiver. Therefore, the first receiver is the strongest and the $N$ th receiver is the weakest [2]. From Lemma 4, we know that there is a cutoff power for each of the strongest $N-1$ receivers, which are denoted as $P_{c 1}, P_{c 2}, \cdots, P_{c(N-1)}$. If the optimal total transmission power is below $P_{c 1}$, all the power is allocated to the strongest receiver and the power allocated to the remaining $N-1$ receivers are zero. If the optimal total

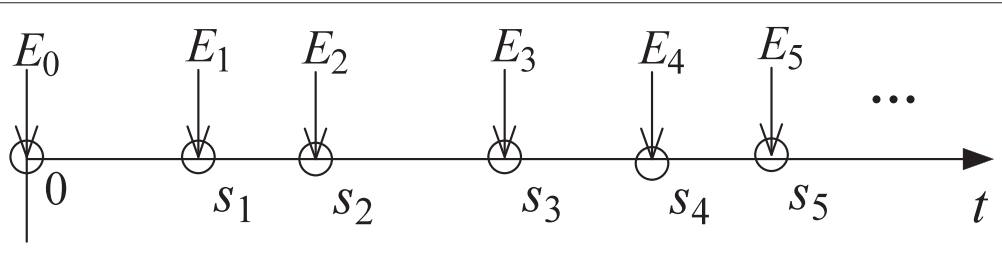

Figure 3 Energy arriving process for the 'whole transmitter'. 
transmission power is higher than $P_{c 1}$, the power allocated to the first receiver is $P_{c 1}$. Then, we check whether the remaining power is below $P_{c 2}$ or not. If the remaining power is higher than $P_{c 2}$, the power $P_{c 2}$ will be allocated to the second strongest receiver. Otherwise, all the remaining power will be allocated to the second strongest receiver and power will not be allocated to the remaining $N-2$ receivers. The rest can be done in the same manner.

From Corollary 1 of Lemma 4, we know that the power for the data transmission to every receiver is either a nonnegative constant sequence or an increasing non-negative sequence.

From Lemma 5, we know that with the optimal power allocation policy, all the data sent to the respective receivers must be finished at the same time.

With these properties and based on the results in [2], the optimal total transmission powers and the cutoff powers are obtained, which are plotted in Figure 4.

\subsection{Proposed power allocation policy}

Aiming at minimizing the transmission completion time, we propose an alternative power allocation policy that performs close to the optimal one but is simpler. The idea is that we heuristically make the transmission rates proportional. This satisfies the properties mentioned in the previous subsection. To illustrate the proposed power allocation policy, we partition the total transmission time into several time slots according to Figure 4. In every time slot, there is no transmitter switching and the transmission rate keeps constant. Next we derive the relationship between the amount of bits to be transmitted and the transmission rates in all time slots. Take the data $B_{a}$ which corresponds to $\mathrm{RX}_{a}$ as an example. The partitioning of time slots and the corresponding transmission rates are shown in Figure 5 . In the first time slot $L_{1}$, the transmission rate for $B_{a}$ is $\underline{r}_{1 a}$. The rate during the next time slot is $\underline{r}_{2 a}$, the transmission completion time is $T_{e}, f$ is the number of the time slots, and $f$ is equal to or greater than the number of switches (when the current working transmitter has energy left at the time the optimal total transmission power changes). The following equation can be easily obtained:

$$
B_{a}=\underline{r}_{1 a} L_{1}+\underline{r}_{2 a} L_{2}+\cdots+\underline{r}_{f a} L_{f} .
$$

For notational simplicity, we continue the derivation at the system with three receivers, which are $\mathrm{RX}_{1}, \mathrm{RX}_{2}$, and $\mathrm{RX}_{3}$. The derivation holds in the case of more receivers. The data to be delivered to the receivers are $B_{1}, B_{2}$, and $B_{3}$. The power allocated to $\mathrm{RX}_{1}, \mathrm{RX}_{2}$, and $\mathrm{RX}_{3}$ are $P_{1}, P_{2}$, and $P_{3}$, respectively. During every time slot, $P_{1}, P_{2}$, and $P_{3}$ are constant. The relationship between the total transmission power and $P_{1}, P_{2}$, and $P_{3}$ is given by

$$
P_{1}+P_{2}+P_{3}=P_{d l} .
$$

From (4), we get the following equations:

$$
\begin{aligned}
& B_{1}=\underline{r}_{11} L_{1}+\underline{r}_{21} L_{2}+\cdots+\underline{r}_{f 1} L_{f}, \\
& B_{2}=\underline{r}_{12} L_{1}+\underline{r}_{22} L_{2}+\cdots+\underline{r}_{f 2} L_{f}, \\
& B_{3}=\underline{r}_{13} L_{1}+\underline{r}_{23} L_{2}+\cdots+\underline{r}_{f 3} L_{f} .
\end{aligned}
$$

We set $\frac{\underline{r}_{q 1}}{\underline{r}_{q 2}}=k_{1}$ and $\frac{\underline{r}_{q 1}}{\underline{r}_{q 3}}=k_{2}\left(k_{1}\right.$ and $k_{2}$ are constants). By substituting them in the first equation of (6), we can get that

$$
\begin{aligned}
& B_{1}=k_{1} \underline{r}_{12} L_{1}+k_{1} \underline{r}_{22} L_{2}+\cdots+k_{1} \underline{r}_{f 2} L_{f}=k_{1} B_{2}, \\
& B_{1}=k_{2} \underline{r}_{13} L_{1}+k_{2} \underline{r}_{23} L_{2}+\cdots+k_{2} \underline{r}_{f 3} L_{f}=k_{2} B_{3} .
\end{aligned}
$$

Then, we obtain the relationship

$$
\frac{B_{1}}{\underline{r}_{q 1}}=\frac{B_{2}}{\underline{r}_{q 2}}=\frac{B_{3}}{\underline{r}_{q 3}} .
$$

Substituting (2) into (8) and combining (5), the power allocation $P_{1}, P_{2}$, and $P_{3}$ in every time slot can be obtained.

\subsection{Transmitter switching policy}

In this subsection, a transmitter switching policy for choosing the suitable transmitter to work will be presented. With the optimal total transmission power and the allocated powers at hand, the transmission completion time can be determined. For a given transmission completion time $T_{e}$, the following propositions are introduced.

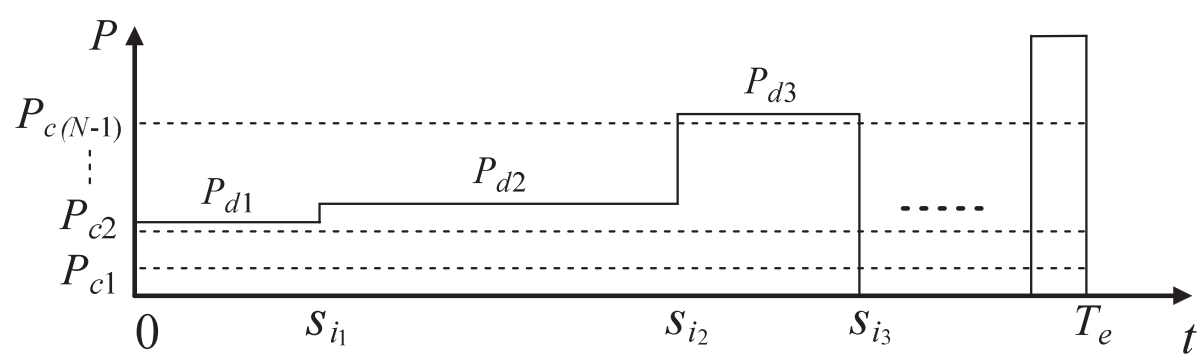

Figure 4 Optimal total transmission power for the 'whole transmitter'. 


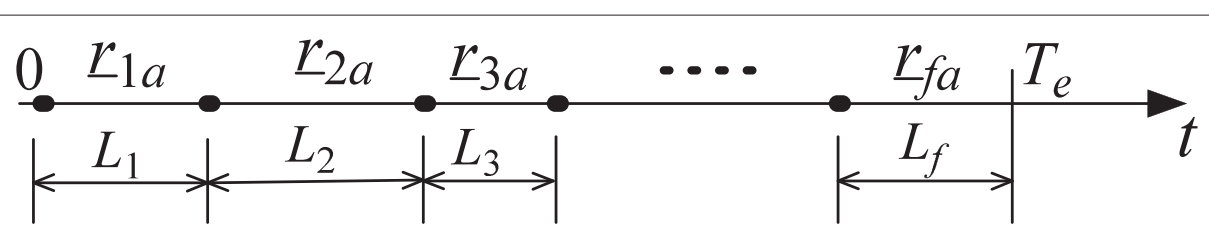

Figure 5 Partitioning of time slots and corresponding transmission rates for the data $\boldsymbol{B}_{\boldsymbol{a}}$.

Proposition 1. The transmitter which harvests energy at its last energy harvesting time before the transmission completion time will turn to work as long as the working transmitter uses up its energy.

Proof. The transmitter which harvests energy at its last energy harvesting time before the transmission completion time is named as full transmitter (which has finished energy harvesting), and the other transmitters are called partial transmitters. We assume that the current working transmitter is $\mathrm{TX}_{a}$ and it sends data with power $P_{d l}$ (as transmitter switching is not affected by power allocation). Moreover, the optimal total transmission power keeps constant over several switches (if there is only one switch during the time the optimal total transmission power keeps constant, the following analysis still holds). The full transmitter $\mathrm{TX}_{b}$ harvests the last energy at the time instant $s_{b e}$ with amount $E_{b e}$, as shown in Figure 6. The total amount of energy available for $\mathrm{TX}_{b}$ is denoted by $E_{b o}$ (energies harvested by $\mathrm{TX}_{b}$ at earlier energy harvesting time may not be used up). The next switching instant is denoted by $s^{\prime}$. For clarity, we still take the system with three transmitters as an example. The analysis can be easily extended to the system with more transmitters. The partial transmitter is denoted by $\mathrm{TX}_{c}$ with the amount of energy $E_{c o}$ at the time instant $s^{\prime}$. There are two possible cases in which the transmitter should work first between $\mathrm{TX}_{b}$ and $\mathrm{TX}_{c}$.
In case 1 , we assume that the partial transmitter $\mathrm{TX}_{c}$ works first. The amount of energy harvested by $\mathrm{TX}_{c}$ during the interval $\left(s^{\prime}, s^{\prime}+t^{*}\right)$ is denoted by $E_{c 1}$, and $t^{*}=$ $\frac{E_{c o}+E_{c 1}}{P_{d l}}$. At the time instant $s^{\prime}+t^{*}, \mathrm{TX}_{b}$ turns to work. The length of the working time slot for $\mathrm{TX}_{b}$ is $t^{\prime}=\frac{E_{b o}}{P_{d l}}$. At the time instant $s^{\prime}+t^{*}+t^{\prime}$, another transmitter turns to work.

In case 2 , we assume that the full transmitter $\mathrm{TX}_{b}$ works first. At the time instant $s^{\prime}+t^{\prime}, \mathrm{TX}_{c}$ turns to work. During the interval $\left(s^{\prime}+t^{*}, s^{\prime}+t^{\prime}+t^{*}+t^{+}\right)$, the amount of energy harvested by $\mathrm{TX}_{c}$ is $E_{c 1^{\prime}}$, and $t^{+}=\frac{E_{c 1^{\prime}}}{P_{d l}}$. It is easy to check that $t^{+} \geq 0$. At the time instant $s^{\prime}+t^{*}+t^{\prime}+t^{+}$, another transmitter turns to work.

The length of the working time slots with two switches in case 2 must be longer than or equal to the one in case 1 . For a given transmission completion time $T_{e}$, the longer working time slot per switch will bring less number of switches. Hence, the full transmitter should work first.

Proposition 2. If there is more than one full transmitter, the working order of the full transmitters does not affect the number of switches.

Proof. When there is more than one full transmitter, we let all of them work earlier than the partial transmitters based on Proposition 1. This prolongs the energy harvesting time for the partial transmitters before they use up their energies. As there is no energy arriving to the full transmitters, which full transmitter turns to work first has

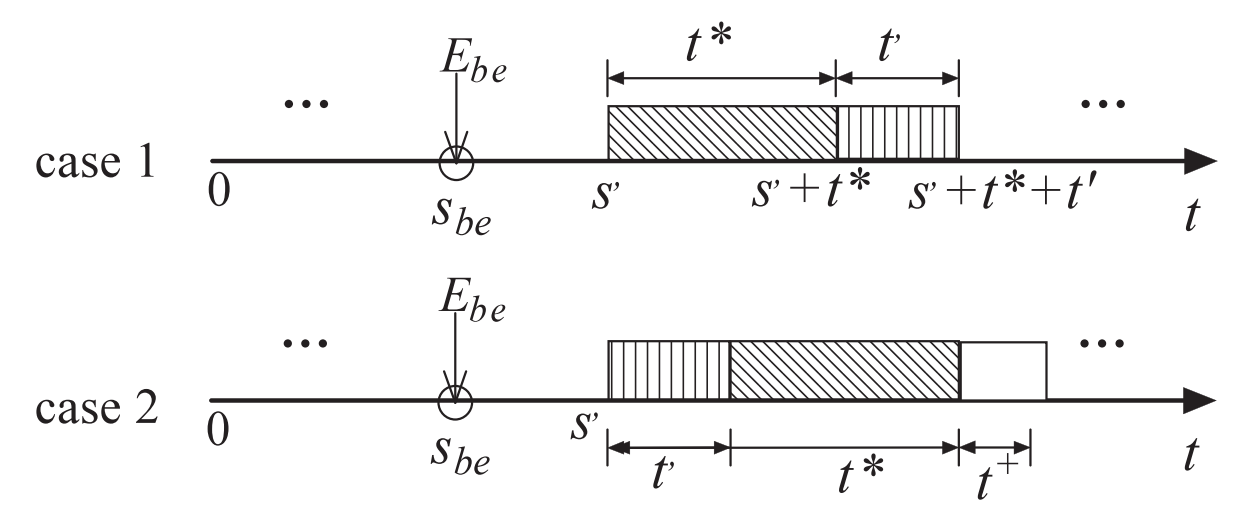

Figure 6 Two cases of working order of the full transmitter and the partial transmitter. 
no influence on the working time slots for the remaining full transmitters. So the working order of the full transmitters has no effect on the partial transmitters and also does not affect the number of switches.

Proposition 3. When no full transmitter exists in the system, the transmitter with the maximum amount of energy available should work first.

Proof. Greater amount of energy brings longer working time with the same transmission power. For a given transmission completion time $T_{e}$, longer working time leads to less number of switches.

With the above propositions, the suitable transmitter can be found. To help understand the use of harvested energy, we take a partial transmitter $\mathrm{TX}_{u}$ as an example, as shown in Figure 7. The use of harvested energy for other transmitters is similar to $\mathrm{TX}_{u}$. In Figure $7, E_{u 0}$ is the amount of energy available in the battery of $\mathrm{TX}_{u}$ at the present time $T$. At the time instant $s_{u w}, \mathrm{TX}_{u}$ harvests energy with amount $E_{u w}$. With the transmission power $P_{d l}$, the amount of energy $E_{u 0}$ can make the transmitter work for a time slot $t_{1}$, where $t_{1}=E_{u 0} / P_{d l}$. During this time slot, if there is new energy arriving, it will be harvested by $\mathrm{TX}_{u}$ and put into use before switching. For example, two energies $E_{u 1}$ and $E_{u 2}$ can be harvested before switching. Then, the new harvested energy $E_{u 1}+E_{u 2}$ will be used to send data for a new time slot $t_{2}=\frac{E_{u 1}+E_{u 2}}{P_{d l}}$. Until the time instant $T+t_{1}+t_{2}$, if there is new energy arriving, it will be harvested and used for keeping $\mathrm{TX}_{u}$ work; otherwise, if there is no new energy arriving, at the time instant $T+t_{1}+t_{2}$, another transmitter will turn to work.

\section{Simulation results}

Numerical simulations are conducted to demonstrate the power allocation policies and the transmitter switching policy. First, the proposed power allocation policy is compared with the optimal power allocation policy. Then, the proposed power allocation policy is compared with some heuristic power allocation policies. Finally, the proposed transmitter switching policy is compared with some heuristic transmitter switching policies.
4.1 Comparison with the optimal power allocation policy We take the energy harvesting communication system with $M=3$ and $N=3$ as an example. The length between two consecutive energy arriving time for $\mathrm{TX}_{1}, \mathrm{TX}_{2}$, and $\mathrm{TX}_{3}$ obeys exponential distribution with parameters $\lambda_{1}=$ $0.01, \lambda_{2}=0.1$, and $\lambda_{3}=1$, respectively. The amount of harvested energy $E_{m w}(\mathrm{~mJ})$ obeys uniform distribution in the interval $(0,0.01),(0,0.02)$, and $(0,0.03)$, respectively. Note that there is no actual model of the distributions of the stochastic energy arriving time and amount of arrived energy yet. We adopt these distributions just for exposition purpose. The analysis in the previous section does not depend on the distributions. The bits to be sent to $\mathrm{RX}_{1}, \mathrm{RX}_{2}$, and $\mathrm{RX}_{3}$ are $B_{1}=70$ bit, $B_{2}=20$ bit, and $B_{3}=10$ bit, respectively. The three transmitters have the same channel parameters as follows: the bandwidth $B o=1 \mathrm{MHz}$; the path loss between $\mathrm{TX}_{m}$ and $\mathrm{RX}_{1}, \mathrm{RX}_{2}$, $\mathrm{RX}_{3}$ is $h_{m 1}=100 \mathrm{~dB}, h_{m 2}=101 \mathrm{~dB}$, and $h_{m 3}=102$ $\mathrm{dB}$, respectively, $m=1,2,3$; the noise power spectral density is $N_{m n}=10^{-19} \mathrm{~W} / \mathrm{Hz}, m=1,2,3, n=1,2,3$. The transmission rates can be written as follows:

$$
\begin{aligned}
& r_{m 1}=\log _{2}\left(1+\frac{P_{1}}{10^{-3}}\right) \text { Mbps } \\
& r_{m 2}=\log _{2}\left(1+\frac{P_{2}}{P_{1}+10^{-2.9}}\right) \text { Mbps } \\
& r_{m 3}=\log _{2}\left(1+\frac{P_{3}}{P_{1}+P_{2}+10^{-2.8}}\right) \text { Mbps. }
\end{aligned}
$$

According to the above simulation parameters, we can get the optimal total transmission powers of the whole transmitter as $P_{d 1}=0.4712 \mathrm{~mW}, P_{d 2}=0.5910 \mathrm{~mW}$, $P_{d 3}=0.6139 \mathrm{~mW}, P_{d 4}=0.6593 \mathrm{~mW}$, and $P_{d 5}=0.7263$ $\mathrm{mW}$. The corresponding time instants are $s_{i_{1}}=0.1691$ $\mathrm{s}, s_{i_{2}}=2.8973 \mathrm{~s}, s_{i_{3}}=7.7806 \mathrm{~s}, s_{i_{4}}=10.7788 \mathrm{~s}$, and $s_{i_{5}}=10.7906 \mathrm{~s}$. With the proposed power allocation policy, until the time instant $10.788761418 \mathrm{~s}, 1,200$ times of harvested energy is consumed by the system, the number of switches is 47 , and all the bits are delivered to their intended receivers. We plot the allocated powers in the top panel of Figure 8. With the proposed power allocation policy, the powers $P_{1}, P_{2}$, and $P_{3}$ remain constant during a time slot and increase at the time instants $s_{i l}$. We also plot the allocated powers under the optimal power allocation

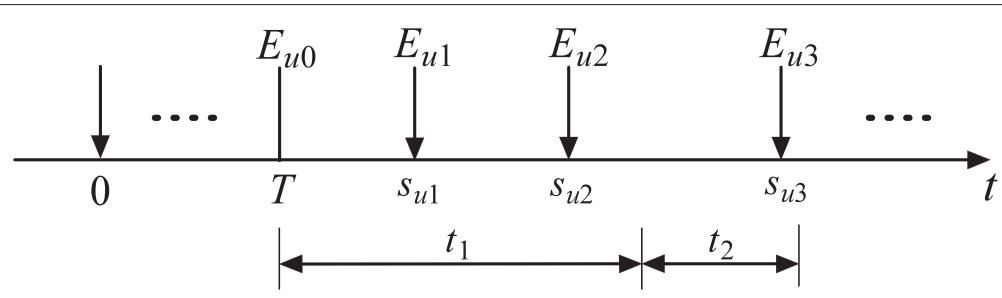

Figure 7 The use of harvested energy for a partial transmitter $\mathrm{TX}_{u}$. 

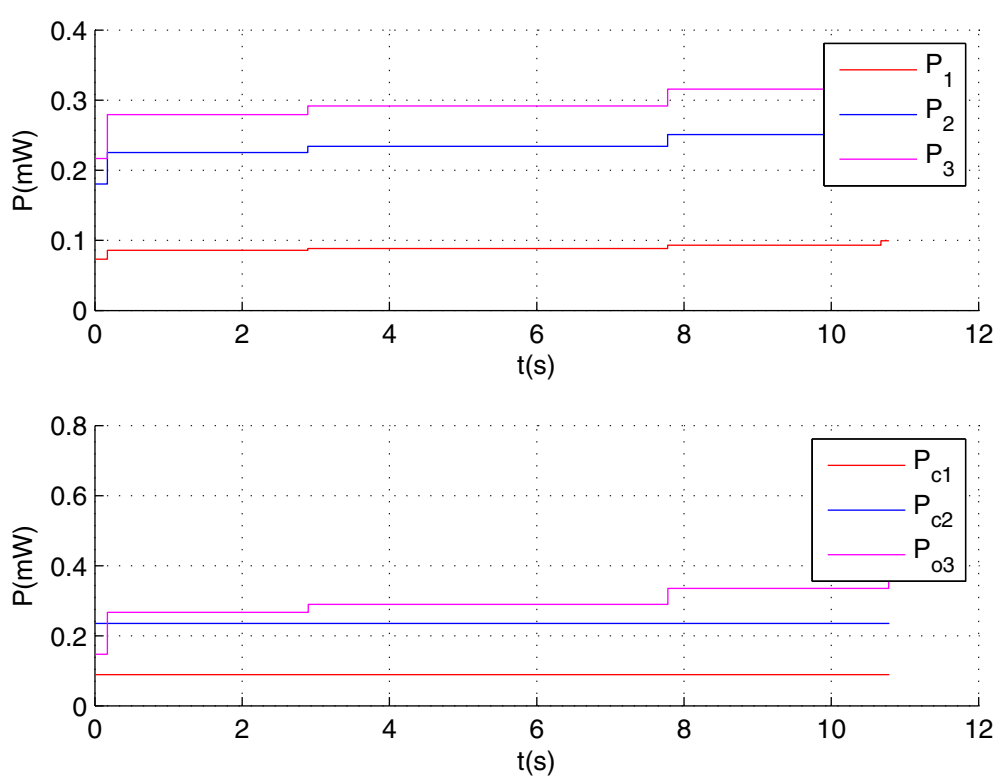

Figure 8 Power allocation under the proposed policy (top) and the optimal policy (bottom).

policy in the bottom panel of Figure 8. The transmission completion time of the optimal power allocation policy is $10.788513518 \mathrm{~s}$. The power allocated to $\mathrm{RX}_{1}$ is a constant $P_{c 1}=0.0888 \mathrm{~W}$, the power allocated to $\mathrm{RX}_{2}$ is also a constant $P_{c 2}=0.2354 \mathrm{~W}$, and the remaining power $P_{o 3}=$ $P_{d l}-P_{c 1}-P_{c 2}$ is allocated to $\mathrm{RX}_{3}$. Because the optimal total transmission power is a constant or an increasing sequence, $P_{o 3}$ changes simultaneously with $P_{1}, P_{2}$, and $P_{3}$.
Even though the transmission completion time under the proposed power allocation policy is $2.4790 \times 10^{-4}$ s longer than the one under the optimal power allocation policy, the relative deviation is $0.04 \%$, which can be neglected.

Moreover, we simulate the effect of multiple of bits on the relative deviation under the power allocation policies, as shown in Figure 9. The base of bits are $B_{1}=7$ bit, $B_{2}=5 \mathrm{bit}$, and $B_{3}=2 \mathrm{bit}$. It is observed that the

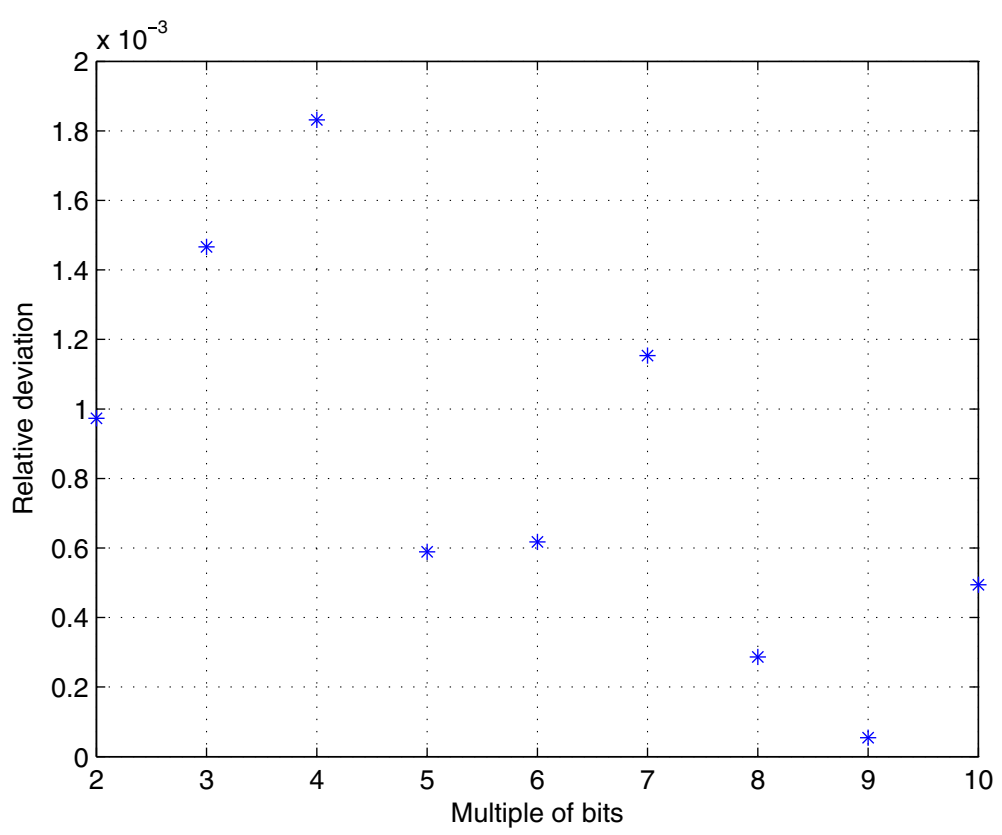

Figure 9 Effect of multiple of bits on relative deviation. 
increase of number of bits may not enlarge the relative deviation, though it prolongs the transmission completion time. Moreover, the relative deviations are small for a moderate amount of bits.

\subsection{Comparison with heuristic power allocation policies}

To show the advantage of the proposed power allocation policy, we compare it with some heuristic power allocation policies as follows:

1. Equal power (EP) policy: In this policy, the optimal total transmission power is equally allocated to the three receivers. When the data transmission intended to a receiver is completed, that receiver is not involved in power allocation.

2. Data ratio (DR) policy: The proposed power allocation policy allocates the optimal total transmission power according to the ratio of the amount of bits to be transmitted and the transmission rate in every time slot. In this policy, the optimal total transmission power is allocated according to the ratio of the amount of bits to be transmitted and the allocated powers in every time slot, which is

$$
\frac{B_{1}}{P_{1}}=\frac{B_{2}}{P_{2}}=\cdots=\frac{B_{N}}{P_{N}} .
$$

When the data transmission intended to a receiver is completed, that receiver is not involved in power allocation.

3. Remaining data ratio (RDR) policy: In this policy, the optimal total transmission power is allocated according to the ratio of the remaining bits and the allocated powers in every time slot, which is

$$
\frac{B_{1}-B_{o 1}}{P_{1}}=\frac{B_{2}-B_{o 2}}{P_{2}}=\cdots=\frac{B_{N}-B_{o N}}{P_{N}}
$$

where $B_{\text {on }}$ is the number of bits that has been sent to $\mathrm{RX}_{n}$ at the previous switching time instant. When the data transmission intended to a receiver is completed, that receiver is not involved in power allocation.

In this subsection, we set $B_{1}=15$ bit, $B_{2}=10$ bit, and $B_{3}=7$ bit. The other parameters are the same as those in Section 4.1. Recall that the energy harvesting processes are stochastic. We take 1,000 independent runs for the same setting and get the average transmission completion time, which are listed in Table 1. The proposed power allocation policy leads to the least average transmission completion time among the policies. The RDR policy allocates the power according to the remaining bits in time, which guarantees that all the data transmissions are completed nearly at the same time. Hence, the average transmission completion time under this policy is the second least. However, it is nearly double of the average
Table 1 Average transmission completion time under the power allocation policies

\begin{tabular}{lc}
\hline Policy & Average transmission completion time (s) \\
\hline EP & 11.93 \\
DR & 6.75 \\
RDR & 6.20 \\
Proposed & 3.46 \\
\hline
\end{tabular}

transmission completion time under the proposed policy. The EP and the DR policies allocated the power in a fixed manner, which cannot guarantee that the data transmissions are completed at the same time or nearly the same time. Thus, these policies lead to longer average transmission completion time.

\subsection{Comparison with heuristic transmitter switching policies}

In this part, we compare the proposed transmitter switching policy with some heuristic ones under the proposed power allocation policy. The simulation parameters are the same as those in Section 4.2. The heuristic transmitter switching policies are given as follows:

1. Energy minimum (EM) policy: In this policy, at every switching time instant, we choose the transmitter with the minimum energy to work.

2. Fixed order 123 (FO123) policy: In this policy, we let the order of switching be fixed: $\mathrm{TX}_{1}$ works first. When it uses up its energy, $\mathrm{TX}_{2}$ turns to work. $\mathrm{TX}_{3}$ works last. When $\mathrm{TX}_{3}$ uses up its energy, a new turn starts again.

3. Fixed order 132 (FO132) policy: This policy is similar to the previous policy, but the turn of switching changes, that is, $\mathrm{TX}_{3}$ works second and $\mathrm{TX}_{2}$ works last.

4. Stochastic switching (SS) policy: In this policy, when a transmitter uses up its energy, we choose another transmitter to work randomly.

We take 10,000 independent runs and get the average number of switches, which are listed in Table 2 . It is seen that the proposed policy leads to the least average

Table 2 Average number of switches under the transmitter switching policies

\begin{tabular}{lc}
\hline Policy & Average number of switches \\
\hline Proposed & 18.43 \\
EM & 20.33 \\
FO123 & 26.08 \\
FO132 & 26.09 \\
SS & 46.39 \\
\hline
\end{tabular}


number of switches among the policies. The EM policy chooses the transmitter with minimum energy, which means that each working time slot is short. Therefore, the number of switches under it must be greater than the one under the proposed policy. Both FO123 and FO132 policies have fixed switching order. Thus, they nearly attain the same average number of switches. The SS policy randomly chooses a transmitter to work, which brings the largest average number of switches. These three heuristic policies do not consider the amount of energy in the battery of transmitters. Their performances must be worse than that under the proposed policy.

\section{Conclusions}

The problem of broadcasting in a communication system with multiple energy harvesting transmitters and multiple receivers has been discussed. To minimize the transmission completion time, we view the transmitters as a whole transmitter, then calculate the optimal total transmission power and reiterate an optimal power allocation policy in our system setup. Moreover, to reduce the complexity of power allocation, a simpler power allocation policy is developed which nearly attains the same transmission completion time with the optimal one and leads to less transmission completion time than some heuristic ones. To enhance the flexibility and robustness of the system, a transmitter switching policy is further developed which leads to less number of switches than some heuristic ones.

\section{Competing interests}

The authors declare that they have no competing interests.

\section{Acknowledgements \\ This research was supported by the National Natural Science Foundation of China $(61162008,61172055)$, the Guangxi Natural Science Foundation (2013GXNSFGA019004), the Open Research Fund of Guangxi Key Lab of Wireless Wideband Communication \& Signal Processing (12103), the Director Fund of Key Laboratory of Cognitive Radio and Information Processing (Guilin University of Electronic Technology), Ministry of Education, China (2013ZRO2), and the Open Research Fund of State Key Laboratory of Networking and Switching Technology (SKLNST-2011-1-01).}

\footnotetext{
Author details

${ }^{1}$ Key Laboratory of Cognitive Radio and Information Processing, Guilin University of Electronic Technology, Ministry of Education, Guilin 541004, China. ${ }^{2}$ School of Automation, Guangdong University of Technology, Guangzhou 510006, China. ${ }^{3}$ School of Electronic and Information Engineering, South China University of Technology, Guangzhou 510641, China.

Received: 30 August 2014 Accepted: 3 November 2014 Published: 12 November 2014

\section{References}

1. F Zhou, H Chen, F Zhao, Transmission scheduling for broadcasting with two energy harvesting switching transmitters. IET Wireless Sensor Syst. 3, 138-144 (2013)

2. J Yang, O Ozel, S Ulukus, Broadcasting with an energy harvesting rechargeable transmitter. IEEE Trans. Wireless Comm. 11, 571-583 (2012)

3. $Y$ Zhang, $\mathrm{S} \mathrm{He}$, J Chen, $Y$ Sun, $X$ Shen, Distributed sampling rate control for rechargeable sensor nodes with limited battery capacity. IEEE Trans. Wireless Comm. 12, 3096-3106 (2013)
}

4. R Yu, Y Zhang, S Gjessing, W Xia, K Yang, Toward cloud-based vehicular networks with efficient resource management. IEEE Network. 27, 48-55 (2013)

5. S Reddy, CR Murthy, Dual-stage power management algorithms for energy harvesting sensors. IEEE Trans. Wireless Comm. 11, 1434-1445 (2012)

6. P Castiglione, O Simeone, E Erkip, T Zemen, Energy management policies for energy-neutral source-channel coding. IEEE Trans. Commun. 60, 2668-2678 (2012)

7. KJ Prabuchandran, SK Meena, S Bhatnagar, Q-learning based energy management policies for a single sensor node with finite buffer. IEEE Wireless Commun. Lett. 2, 82-85 (2013)

8. N Michelusi, K Stamatiou, M Zorzi, Transmission policies for energy harvesting sensors with time-correlated energy supply. IEEE Trans. Commun. 61, 2988-3001 (2013)

9. H Besbes, G Smart, D Buranapanichkit, C Kloukinas, Analytic conditions for energy neutrality in uniformly-formed wireless sensor networks. IEEE Trans. Wireless Commun. 12, 4916-4931 (2013)

10. N Michelusi, L Badia, R Carli, L Corradini, M Zorzi, Energy management policies for harvesting-based wireless sensor devices with battery degradation. IEEE Trans. Commun. 61, 4934-4947 (2013)

11. B Devillers, D Gunduz, A general framework for the optimization of energy harvesting communication systems with battery imperfections J. Comm. Network. 14, 130-139 (2012)

12. CK Ho, R Zhang, Optimal energy allocation for wireless communications with energy harvesting constraints. IEEE Trans. Signal Process. 60, 4808-4818 (2012)

13. O Ozel, S Ulukus, Achieving AWGN capacity under stochastic energy harvesting. IEEE Trans. Inform. Theor. 58, 6471-6483 (2012)

14. S Luo, R Zhang, TJ Lim, Optimal save-then-transmit protocol for energy harvesting wireless transmitters. IEEE Trans. Wireless Comm. 12, 1196-1207 (2013)

15. N Tekbiyik, T Girici, E Uysal-Biyikoglu, K Leblebicioglu, Proportional fair resource allocation on an energy harvesting downlink. IEEE Trans. Wireless Comm. 12, 1699-1711 (2013)

16. P Blasco, D Gunduz, M Dohler, A learning theoretic approach to energy harvesting communication system optimization. IEEE Trans. Wireless Comm. 12, 1872-1882 (2013)

17. CHuang, R Zhang, S Cui, Throughput maximization for the gaussian relay channel with energy harvesting constraints. IEEE J. Sel. Area. Comm. 31, 1469-1479 (2013)

18. J Xu, R Zhang, Throughput optimal policies for energy harvesting wireless transmitters with non-ideal circuit power. IEEE J. Sel. Area. Comm. 32, 322-332 (2014)

19. N Hassan ul, C Yuen, S Saeed, Z Zhang, Power control for sum rate maximization on interference channels under sum power constraint. IEEE Trans. Veh. Tech. (2014)

20. J Yang, S Ulukus, Optimal packet scheduling in an energy harvesting communication system. IEEE Trans. Commun. 60, 220-230 (2012)

21. KTutuncuoglu, A Yener, Optimum transmission policies for battery limited energy harvesting nodes. IEEE Trans. Wireless Commun. 11, 1180-1189 (2012)

22. MA Antepli, E Uysal-Biyikoglu, H Erkal, Optimal packet scheduling on an energy harvesting broadcast link. IEEE J. Sel. Area. Comm. 29, 1721-1731 (2011)

23. H Erkal, FM Ozcelik, E Uysal-Biyikoglu, Optimal offline broadcast scheduling with an energy harvesting transmitter. EURASIP J. Wireless Commun. Netw. (2013)

24. O Ozel, J Yang, S Ulukus, Optimal broadcast scheduling for an energy harvesting rechargeable transmitter with a finite capacity battery. IEEE Trans. Wireless Comm. 11, 2193-2203 (2012)

25. O Ozel, K Tutuncuoglu, J Yang, S Ulukus, A Yener, Transmission with energy harvesting nodes in fading wireless channels: optimal policies. IEEE J. Sel. Area. Comm. 29, 1732-1743 (2011)

26. J Yang, S Ulukus, Optimal packet scheduling in a multiple access channe with energy harvesting transmitters. J. Comm. Network. 14, 140-150 (2012)

27. O Ozel, J Yang, S Ulukus, Optimal transmission schemes for parallel and fading Gaussian broadcast channels with an energy harvesting rechargeable transmitter. Comput. Comm. 36, 1360-1372 (2013) 
28. KTutuncuoglu, A Yener, Sum-rate optimal power policies for energy harvesting transmitters in an interference channel. J. Comm. Network. 14, 151-161 (2012)

29. M Kashef, A Ephremides, Optimal packet scheduling for energy harvesting sources on time varying wireless channels. J. Comm. Network. 14, 121-129 (2012)

30. B Gurakan, O Ozel, J Yang, S Ulukus, Energy cooperation in energy harvesting communications. IEEE Trans. Comm. 61, 4884-4898 (2013)

31. FM Ozcelik, G Uctu, E Uysal-Biyikoglu, Minimization of transmission duration of data packets over an energy harvesting fading channel. IEEE Comm. Lett. 16, 1968-1971 (2012)

32. H Chen, F Zhou, J Cai, F Zhao, Q He, Geometric projection-based switching policy for multiple energy harvesting transmitters. http://arxiv. org/abs/1405.4642

\section{Submit your manuscript to a SpringerOpen ${ }^{\circ}$ journal and benefit from:}

- Convenient online submission

- Rigorous peer review

- Immediate publication on acceptance

- Open access: articles freely available online

- High visibility within the field

- Retaining the copyright to your article 